\title{
Structural, spectroscopic and catalytic activity studies on glutathione reductase reconstituted with FAD analogues
}

\author{
Ulrich ERMLER $^{1}$, Sandro GHISLA ${ }^{2}$, Vincent MASSEY ${ }^{3}$ and Georg E. SCHULZ ${ }^{1}$ \\ 1 Institut für Organische Chemie und Biochemie der Universität, Freiburg im Breisgau, Federal Republic of Germany \\ ${ }^{2}$ Fakultät Biologie der Universität, Konstanz, Federal Republic of Germany \\ 3 Department of Biological Chemistry, University of Michigan, Ann Arbor, USA
}

(Received January 5, 1991) - EJB 910051

FAD-modified human glutathione reductases were reconstituted from apoenzyme using the FAD analogues 6-SH-FAD, 6-SCN-FAD, 6-OH-FAD, 6- $\mathrm{NH}_{2}-\mathrm{FAD}$ and 8-OH-FAD. The catalytic activities of the modified enzymes were substantially lower than for the native enzyme. All five species could be crystallized, but only those containing 6-SH-FAD, 6-OH-FAD and 6- $\mathrm{NH}_{2}-\mathrm{FAD}$ yielded crystals that could be analyzed. $\mathrm{X}$-ray analyses and structural refinements were performed at $0.27 \mathrm{~nm}$ and $0.30 \mathrm{~nm}$ resolution resulting in $R$ factors around $13.5 \%$. The crystal structures showed the additional non-hydrogen atoms and small conformational changes of the polypeptide that were obviously induced by the substituents of the FAD analogues. The observed changes together with spectroscopic and activity data permit some conclusions about the chemical nature of the substituents.

The flavoenzyme glutathione reductase catalyzes the reduction of oxidized glutathione at the expense of NADPH. The human enzyme is a homodimer of two $52.4-\mathrm{kDa}$ subunits with one FAD/subunit. It is structurally and functionally well known $[1-3]$; its crystal structure has been established at $0.154 \mathrm{~nm}$ resolution [4]. Numerous substrate- and ligandbinding studies were performed in order to elucidate enzyme catalysis by correlating structural with kinetic data [5-7]. Recently, these experiments were supplemented by site-directed mutations of the glutathione reductase species from Escherichia coli [8].

For studying flavin-polypeptide interactions, FAD analogues carrying modifications at the isoalloxazine ring have been used as substitutes for native FAD [9]. Human glutathione reductase is a particularly good model system for such experiments, because its structure is precisely known and its apoenzyme is reasonably stable and binds various $F A D$ analogues. Some FAD-modified glutathione reductases have already been studied with respect to their spectroscopic and catalytic properties [10 - 12]. Here, we analyze FAD-modified glutathione reductases by X-ray diffraction and attempt to correlate structural with spectroscopic and catalytic data.

Correspondence to G. E. Schulz, Institut für Organische Chemie und Biochemie der Universität, Albertstraße 21, W-7800 Freiburg i. Br., Federal Republic of Germany

Abbreviations. GR(6-SH-FAD), the FAD-modified enzyme glutathione reductase from human erythrocytes as reconstituted from the apoenzyme and 6-SH-FAD (the same nomenclature is used for the other FAD analogues); $F_{\text {mod, obs }}$, observed structure factor amplitudes for crystalline glutathione reductase reconstituted with FAD analogues; $F_{6 \mathrm{OH} \text { obs }}, F_{6 \mathrm{SH} \text {, obs }}$ and $F_{6 \mathrm{NH} 2 \text {,obs }}$ indicate particular modifications; $F_{\text {nat, obs }}$, observed structure factor amplitudes for the native enzyme; $\alpha_{\text {nat, calc }}$, calculated structure factor phases for the native enzyme.

Enzyme. NADPH:glutathione oxidoreductase (EC 1.6.4.2).

\section{MATERIALS AND METHODS}

In our experiments we used the FAD analogues 6-SHFAD [13], 6-SCN-FAD [13], 6-OH-FAD [14], 6- $\mathrm{NH}_{2}$-FAD [15] and 8-OH-FAD [16] all of which are specified in Fig. 1. Glutathione reductase was isolated from human erythrocytes as described by Krohne-Ehrich et al. [17]. The apoenzyme was obtained by saturating a holoenzyme solution with $\left(\mathrm{NH}_{4}\right)_{2} \mathrm{SO}_{4}$ at $4{ }^{\circ} \mathrm{C}$ and $\mathrm{pH} 3.0$ [18]. This leads to precipitation and release of FAD. The precipitate was washed several times. Because of the extreme $\mathrm{pH}$ conditions this procedure was finished as fast as possible, usually within $90 \mathrm{~min}$. The FADfree precipitate was dissolved and stored in $100 \mathrm{mM}$ Tris/ $\mathrm{HCl}, 10 \mathrm{mM}$ EDTA, pH 8.7, at concentrations of $4-7 \mathrm{mg}$ apoenzyme $/ \mathrm{ml}$. The protein concentrations were determined photometrically using $A_{280}=1.0$ as the absorption coefficient for $1 \mathrm{mg}$ apoenzyme/ml [19].

The enzyme was reconstituted by adding a slight excess of the respective FAD analogue to the apoenzyme. After incubating for $1 \mathrm{~h}$ at room temperature to allow complete binding of the FAD analogue, the solution of the reconstituted enzyme was centrifuged using an Amicon Centricon 10 ultrafilter to remove excess FAD analogue and low-molecular-<smiles>[Y]c1cc2c(c([X])c1C)N=C1C(=O)NC(=O)N=C1[N+]2[R]</smiles>

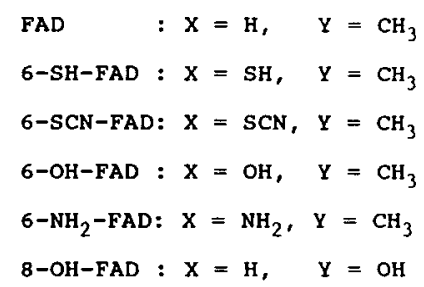

Fig. 1. Isoalloxazine moiety of FAD showing the modified positions. For all FAD analogues $\mathrm{R}$ is ribityl-5'-ADP, as in native FAD 
mass contaminants. In the same manner, the buffer was changed and the enzyme was concentrated for crystallization.

The spectral studies were performed in storage buffer consisting of $100 \mathrm{mM}$ potassium phosphate, $200 \mathrm{mM} \mathrm{KCl,} 1 \mathrm{mM}$ EDTA, $14 \mathrm{mM}$ mercaptoethanol, pH 7.0. For 6-SCN-FAD we used the same procedures, but avoided mercaptoethanol throughout. The enzyme activity was measured under standard conditions [17] in $100 \mathrm{mM}$ potassium phosphate, $200 \mathrm{mM} \mathrm{KCl}, 1 \mathrm{mM}$ EDTA, $0.5 \mathrm{mg} / \mathrm{ml}$ bovine serum albu$\min , \mathrm{pH} 6.9$, at $25^{\circ} \mathrm{C}$.

The reconstituted FAD-modified enzymes were crystallized at $20^{\circ} \mathrm{C}$ using the hanging-drop method under the same conditions employed previously for the native enzyme [20]. The drop consisted of $0.8 \mathrm{M}\left(\mathrm{NH}_{4}\right)_{2} \mathrm{SO}_{4}, 0.1 \mathrm{M}$ potassium phosphate, $\mathrm{pH} 7.0$, with a protein concentration of about $16 \mathrm{mg} / \mathrm{ml}$. The reservoir was filled with $1 \mathrm{ml} 1.2 \mathrm{M}$ $\left(\mathrm{NH}_{4}\right)_{2} \mathrm{SO}_{4}, 0.1 \mathrm{M}$ potassium phosphate, $\mathrm{pH}$ 7.0. Crystallization was also achieved (often with better results) without the buffer. The crystals could be stored for months in $1.5 \mathrm{M}$ $\left(\mathrm{NH}_{4}\right)_{2} \mathrm{SO}_{4}, 0.1 \mathrm{M}$ potassium phosphate, $\mathrm{pH}$ 7.0. The same buffer was used for the X-ray analyses.

$\mathrm{X}$-ray data were collected on a modified four-circle diffractometer [21] and processed to obtain the structure factor amplitudes $F_{\text {mod,obs. }}$ Taking the observed structure factor amplitudes $F_{\text {nat, obs }}$ and the calculated phases $\alpha_{\text {nat, calc }}$ of the native enzyme from [4], a $\left(F_{\text {mod, obs }}-F_{\text {nat, obs }}\right) \cdot \exp i \alpha_{\text {nat, calc }}$ differenceFourier map was calculated that showed the structural changes caused by the modifications of FAD. Electron densities and models were visualized on a graphics system (model PS330, Evans \& Sutherland, USA) using the program FRODO [22]. For structural refinement of the 6-SH-FADmodified enzyme [GR(6-SH-FAD)] we used program TNT [23]. When the more convenient program XPLOR [24] became available, it was applied for refining the 6-OH-FAD- and 6- $\mathrm{NH}_{2}-\mathrm{FAD}$-modified glutathione reductases, GR(6-OHFAD) and $\mathrm{GR}\left(6-\mathrm{NH}_{2}-\mathrm{FAD}\right)$, respectively.

\section{RESULTS}

\section{Spectral and catalytic properties}

The FAD-modified glutathione reductases can be characterized by their absorption spectra $[12,14-16,25,26]$. The measured $\lambda_{\max }$ values of the main absorption bands of the bound FAD analogues are given in Table 1. On binding to the apo form of the enzyme, the $\lambda_{\max }$ values of the FAD analogues increased, usually in the order of $10 \mathrm{~nm}$. However, no $\lambda_{\max }$ shift occurred with 6-OH-FAD.

The analogue 6-SH-FAD can be deprotonated at the substituent $S 6 \alpha$ (i.e. sulfur bound to $C 6$, see Fig. 1). On dissociation, an electron pair of $S 6 \alpha$ conjugates with the isoalloxazine nucleus giving rise to an absorption band around $650 \mathrm{~nm}$. Using this absorption band, the $\mathrm{p} K$ value of $6-\mathrm{SH}$ FAD has been determined as 5.9 in the free state [13], and as $<5.0$ when bound to glutathione reductase [11]. The electronic and spectral properties of 6-OH-FAD are similar to those of $6-\mathrm{SH}-\mathrm{FAD}$; it has a similar band around $650 \mathrm{~nm}$. The $\mathrm{p} K$ value of $6-\mathrm{OH}-\mathrm{FAD}$ has been determined as 7.1 in the free state $[10,14]$. We have now measured a $\mathrm{p} K$ of $5.6 \pm 0.5$ for the bound analogue in GR(6-OH-FAD). Given these $\mathrm{p} K$ values, the enzyme-bound 6-OH-FAD and 6-SH-FAD analogues are predominantly in their anionic state at the $\mathrm{pH} 7.0$ of the X-ray analyses.

The increased acidic nature of enzyme-bound 6-SH-FAD and $6-\mathrm{OH}-\mathrm{FAD}$ as compared to the free state must result from
Table 1. Spectral and catalytic properties of glutathione reductase reconstituted with FAD analogues

All data were obtained at $\mathrm{pH} 7.0$ in the buffers given in the text. The catalytic activity is defined as [(specific activity of FAD-modified enzyme) - (specific activity of apoenzyme)]/(specific activity of native enzyme). The error for the 6-substituted FAD analogues is $\pm 1 \%$ and that for $8-\mathrm{OH}-\mathrm{FAD}$ is $\pm 2 \%$ relative catalytic activity, indicating that GR(8-OH-FAD) could be inactive. The larger error for 8-OH-FAD relates to the lower binding affinity of this analogue

\begin{tabular}{lll}
\hline Enzyme-bound FAD & $\begin{array}{l}\lambda_{\max } \text { of main } \\
\text { absorption band }\end{array}$ & $\begin{array}{l}\text { Relative catalytic } \\
\text { activity }\end{array}$ \\
\hline FAD & $n m$ & $\%$ \\
6-SH-FAD & 463 & 100 \\
6-SCN-FAD & 449 & 3 \\
6-OH-FAD & 452 & 0 \\
6-NH & 421 & 0 \\
8-OH-FAD & 442 & 5 \\
\hline
\end{tabular}

a stabilization of the anionic charge within the protein. In 6-SH-FAD and 6-OH-FAD the negative charge is partially located at the substituent atoms $\mathrm{S} 6 \alpha$ and $\mathrm{O} 6 \alpha$ and partially in the isoalloxazine nucleus, where it is enhanced in the region around $\mathrm{N} 1$ and $\mathrm{O} 2 \alpha$. A partial negative charge at $\mathrm{O} 6 \alpha$ and S6 $\alpha$ is stabilized by the contacting positively charged amino group of Lys66. A partial negative charge around $\mathrm{N} 1$ and $\mathrm{O} 2 \alpha$ is stabilized by the favorably oriented amide dipoles of the $\alpha$-helix of amino acid residues $339-354$ that contacts $\mathrm{O} 2 \alpha$ [27].

For $6-\mathrm{NH}_{2}-\mathrm{FAD}$ in the free state, no spectral changes, and thus no ionization, occur in the physiological $\mathrm{pH}$ range. There are two measurable $\mathrm{p} K$ values, the first one around 1 where $N 6 \alpha$ accepts a proton, and the second one around 10 where $\mathrm{N} 3$ loses a proton [28]. The $\mathrm{p} K$ for the deprotonation of the 6- $\mathrm{NH}_{2}$ group is greater than 13 [28]. As a consequence, the $6-\mathrm{NH}_{2}$ substituent has no formal charge at $\mathrm{pH} 7.0$ of the $\mathrm{X}$-ray analysis. Since the lone electron pair of $\mathrm{N} 6 \alpha$ conjugates with the isoalloxazine, nucleus, however, N6 $\alpha$ carries a partial positive charge.

The catalytic activities of the modified glutathione reductases are also given in Table 1 . All of them are appreciably lower than the activity of the native enzyme. Due to the error margins, the activities of GR(6-SCN-FAD) and GR(6-OHFAD) could be as high as $1 \%$ of that of the native enzyme and the activity of GR(8-OH-FAD) could be essentially zero.

\section{Structural studies}

Crystallography. Crystals could be obtained from human glutathione reductase reconstituted with all five FAD analogues. Crystals suitable for X-ray diffraction, however, grew only from GR(6-SH-FAD), GR(6-OH-FAD) and GR(6$\left.\mathrm{NH}_{2}-\mathrm{FAD}\right)$. The enzymes GR(6-SCN-FAD) and GR(8-OHFAD) could only be crystallized up to sizes of 250 $\times 150 \times 70 \mu^{3}$ and $200 \times 100 \times 100 \mu \mathrm{m}^{3}$, respectively, which are too small for reasonable data collection on a diffractometer. The crystallization of GR(8-OH-FAD) was complicated by the weak binding of this analogue to the apoenzyme, which may have given rise to inhomogeneous protein ensembles. The relatively low binding constant of 8 $\mathrm{OH}-\mathrm{FAD}$ as compared to FAD was discovered when $8-\mathrm{OH}-$ FAD was slowly washed out on concentrating GR(8-OHFAD) in the Centricon tube. 


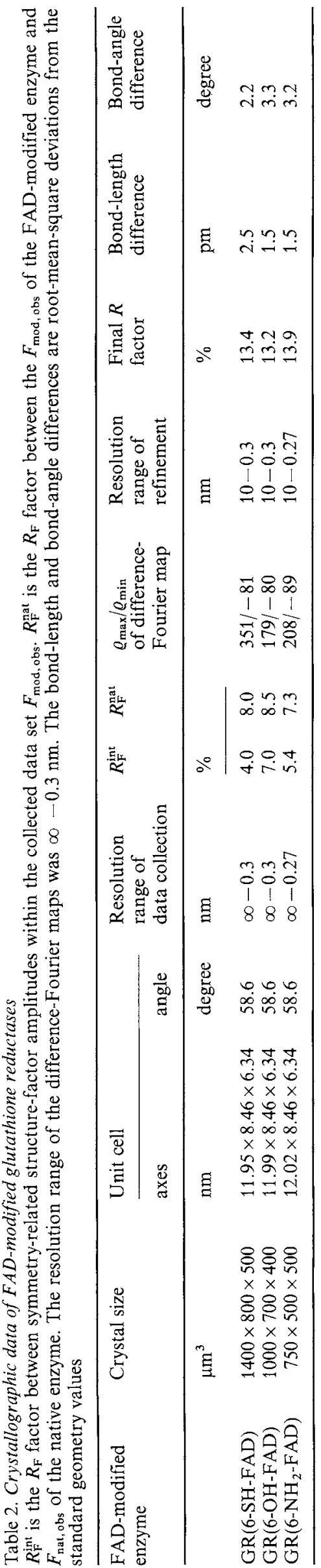

All three analyzed FAD-modified human glutathione reductases crystallized in space group B2 and are isomorphous with the native enzyme crystals (Table 2). Data were collected up to $0.27 \mathrm{~nm}$ resolution. The structural refinements converged at $R$ factors around $13.5 \%$ with good geometries (Table 2). The quality of the structure analyses is also demonstrated in the maxima of the difference-Fourier maps of GR(6$\mathrm{SH}-\mathrm{FAD})$ and GR(6-OH-FAD). These maxima are at the S6 $\alpha$ and $\mathrm{O} 6 \alpha$ positions, and their heights (Table 2) correlate well with the number of electrons of sulfur and oxygen. At the given resolution and the achieved good $R$ factors, the general accuracy of the atomic coordinates can be expected to be $0.03 \mathrm{~nm}$.

In the native enzyme there is a cavity around the $\mathrm{C} 6$ atom of isoalloxazine [4]. This hole extends over a distance of about $0.5 \mathrm{~nm}$ in the direction of the C6-S6 $\alpha, \mathrm{C} 6-\mathrm{O} 6 \alpha$ and C6-N6 $\alpha$ bonds of the modified flavins. Given this free space, the incorporation of the additional 6-SH, 6-OH and 6- $\mathrm{NH}_{2}$ groups caused only small conformational changes of the polypeptide. For the most part, these changes occurred at residues Gly62, Cys63, Lys66 and Glu201, which surround the substituents at C6. It should be noted that the environment of the $\mathrm{C} 6$ atom is polar, which in conjunction with the available space explains the high binding constants of FAD analogues that carry a small polar group at C6. Since the obtained resolutions of the $\mathrm{X}$-ray analyses are merely $0.27 \mathrm{~nm}$ and $0.30 \mathrm{~nm}$, the quantification of these shifts is limited by the residual error.

The structure of $G R(6-S H-F A D)$. The highest peak in the difference-Fourier map between GR(6-SH-FAD) and native glutathione reductase exceeds the second highest peak by a factor of four. As shown in Fig. 2, it corresponds to the sulfur of 6-SH-FAD. The 'observed' C6-S6 $\alpha$ bond length was taken as the distance between the density maximum of the difference-Fourier map and the position of the $\mathrm{C} 6$ atom after the refinement. In this way, the observed bond length becomes largely independent of the respective bond length used in the refinement. The observed C6-S6 $\alpha$ bond length was $0.16 \mathrm{~nm}$, which is slightly, but not significantly, shorter than a double bond $(0.17 \mathrm{~nm})$ and a single bond $(0.18 \mathrm{~nm})$ [29].

Since the $\mathrm{p} K$ value of $6-\mathrm{SH}-\mathrm{FAD}$ is lower than 5.0 when bound to glutathione reductase [11], the FAD analogue should be present in its anionic state with deprotonated $S 6 \alpha$. Presumably, the negative charge at $S 6 \alpha$ is partially delocalized into the flavin-ring system, where it is most likely concentrated around $\mathrm{N} 1$ and $\mathrm{O} 2 \alpha$ and stabilized by the helix of amino acid residues $339-354$. This is consistent with the observed C6S6 $\alpha$ bond being on the shorter side.

The difference-Fourier map in Fig. 2 shows only smallish densities apart from the added S6 $\alpha$. These are at Gly62 and at the redox active disulfide bridge Cys58-Cys63. Using the refined coordinates, these difference densities can be translated into shifts. Accordingly, the new substituent $\mathrm{S} 6 \alpha$ has increased the distance between Gly62 and the isoalloxazine by $0.03 \mathrm{~nm}$, and it has caused the disulfide to move by $0.02 \mathrm{~nm}$ in a direction parallel to the isoalloxazine plane. The coordinates indicate further that the salt bridge Lys66-NZ-Glu201-OE2 is shifted by about $0.01 \mathrm{~nm}$ toward the $\mathrm{C} 6$ atom, which is well within the limits of error and therefore not significant. The resulting distances $\mathrm{S} 6 \alpha \ldots . \mathrm{NZ}, \mathrm{S} 6 \alpha \ldots \mathrm{OE} 2$ and $\mathrm{NZ} \ldots \mathrm{OE} 2$ are $0.30 \mathrm{~nm}, 0.34 \mathrm{~nm}$ and $0.29 \mathrm{~nm}$, respectively. The short distance between $S 6 \alpha$ and NZ points to a hydrogen bond involving a thiolate.

The structure of GR(6-OH-FAD). The highest peak in the $\left(F_{6 \mathrm{OH}, \text { obs }}-F_{\text {nat, obs }}\right) \cdot \exp i \alpha_{\text {nat, calc }}$ difference-Fourier map exceeds the next highest peak by a factor of two. It is caused 

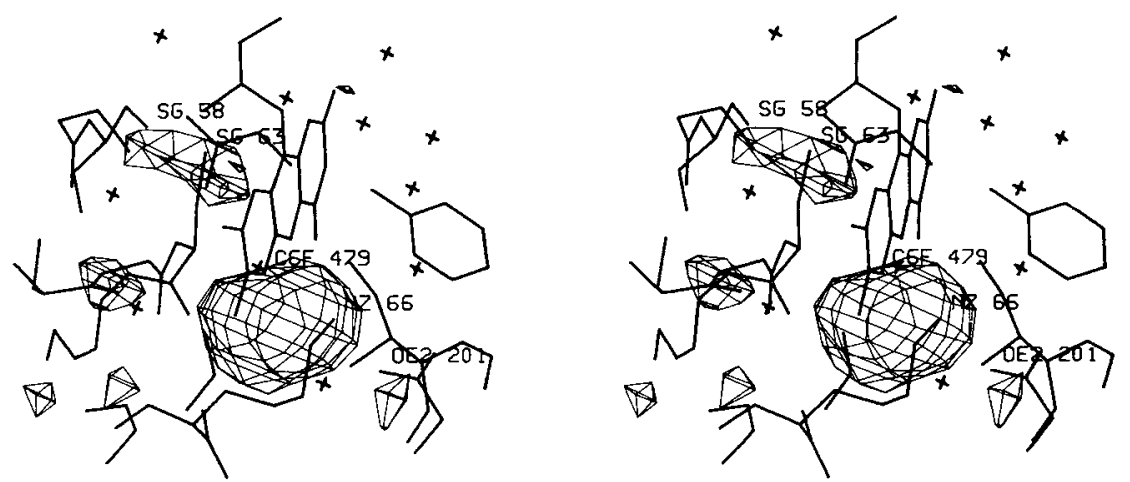

Fig. 2. Difference-Fourier map of glutathione reductase containing 6-SH-FAD calculated from $\left(\mathrm{F}_{6 S H . \text { obs }}-\mathrm{F}_{\text {nat.obs }}\right) \cdot$ expi $\alpha_{\text {nat, calc }} \cdot \mathrm{For}$ clarity, only positive density is given. The contours are drawn at $15 \%$ of the electron-density maximum of this map. The model is centered at the $S 6 \alpha$ atom. Part of the FAD analogue together with chain segments around Cys58, Cys63, Lys66 and Glu201 (all labelled) is depicted, as well as parts of Tyr197, Ile198 and Arg291 (not labelled); (+) water molecules
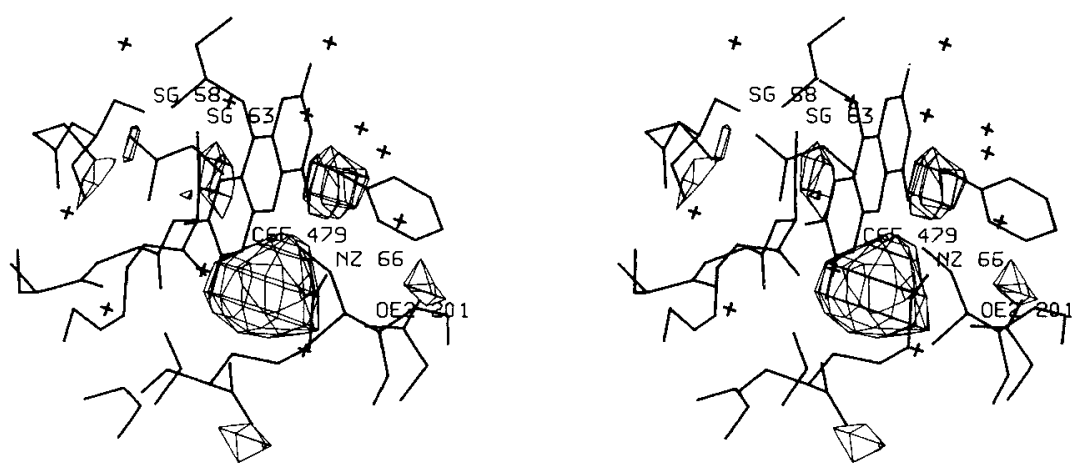

Fig. 3. Difference-Fourier map of glutathione reductase containing $6 . \mathrm{OH}-\mathrm{FAD}$ calculated from $\left(\mathrm{F}_{60 \mathrm{H}, \text { obs }}-\mathrm{F}_{\text {nat.obs }}\right) \cdot$ expi $\alpha_{\text {nat, calc. }}$. For clarity, only positive density is given. The contours are drawn at $28 \%$ of the electron-density maximum of this map. The model is centered at the O6 $\alpha$ atom. Part of the FAD analogue together with chain segments around Cys58, Cys63, Lys66 and Glu201 (all labelled) is depicted, as well as parts of Tyr197, Ile198 and Arg291 (not labelled); (+) water molecules
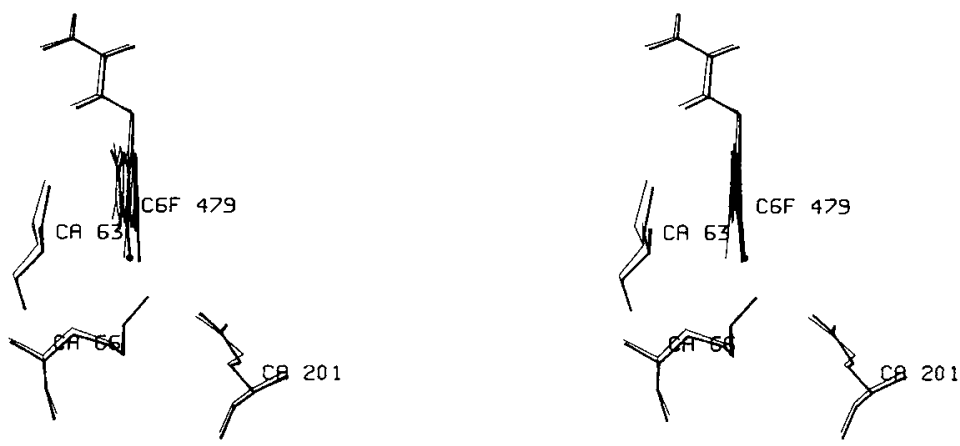

Fig. 4. Structural differences between glutathione reductase containing 6-OH-FAD (thick lines) and the native enzyme (thin lines). Atom O6 is marked by a dot. Depicted is a part of FAD together with residues Cys63, Lys66 and Glu201

by the additional oxygen $\mathrm{O} 6 \alpha$ (Fig. 3). In the same manner as with 6-SH-FAD, the $\mathrm{C} 6-\mathrm{O} 6 \alpha$ bond length was determined as $0.12 \mathrm{~nm}$, which corresponds to a double bond and is smaller than a single bond [29]. The short C6-O6 $\alpha$ bond is consistent with a deprotonated $\mathrm{O} 6 \alpha$, the negative charge of which is delocalized towards the $\mathrm{N} 1-\mathrm{O} 2 \alpha$ region of the isoalloxazine, where it is stabilized by the helix of amino acid residues $339-$ 354 [27]. The deprotonation of $\mathrm{O} 6 \alpha$ at $\mathrm{pH} 7.0$ of the X-ray analysis is in agreement with the measured $\mathrm{p} K$ value of $5.6 \pm 0.5$ for $6-\mathrm{OH}-\mathrm{FAD}$ bound to glutathione reductase.

Other remarkable difference-Fourier peaks are close to $\mathrm{O} 4 \alpha$ and $\mathrm{C} 4 \mathrm{a}$ of isoalloxazine (Fig. 3). In the refinement one of these peaks corresponded to an appreciable shift of $04 \alpha$ caused by a tilt of the isoalloxazine by about $5^{\circ}$ (Fig. 4). The other peak relates to the disulfide bridge which moves together with the adjacent isoalloxazine atoms by about $0.03 \mathrm{~nm}$, keeping the distance constant (Fig. 4).

The refined coordinates indicate further that the tilt of the isoalloxazine has moved the $\mathrm{C} 6$ atom by about $0.04 \mathrm{~nm}$ toward the salt bridge Lys66-NZ-Glu201-OE2. The resulting distances $\mathrm{O} 6 \alpha \ldots \mathrm{NZ}, \mathrm{O} 6 \alpha \ldots \mathrm{OE} 2$ and NZ...OE2 are $0.29 \mathrm{~nm}$, $0.35 \mathrm{~nm}$ and $0.29 \mathrm{~nm}$, respectively, making a partial negative charge at $06 \alpha$ rather likely. Obviously, $06 \alpha$ forms such a strong hydrogen bond to Lys66-NZ that the whole 

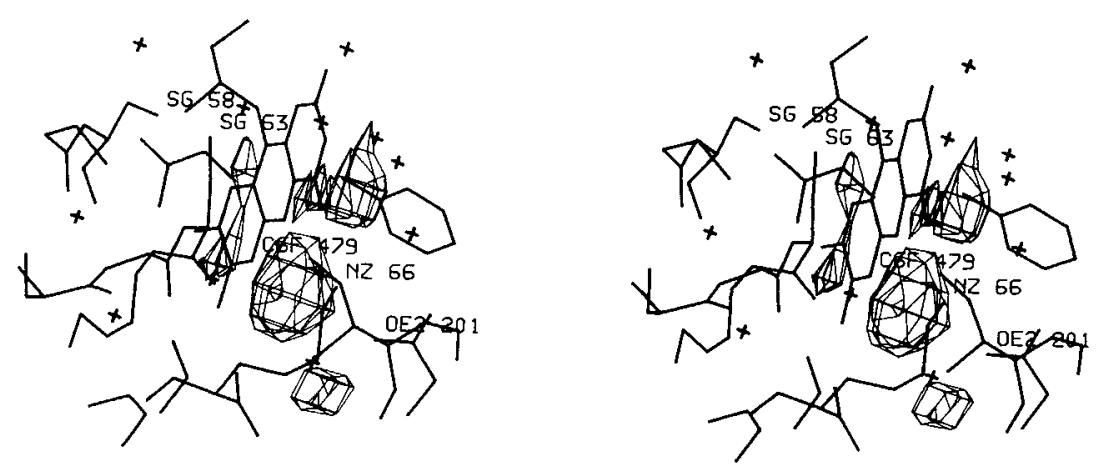

Fig. 5. Difference-Fourier map of glutathione reductase containing $6-\mathrm{NH}_{2}-\mathrm{FAD}$ calculated from $\left(\mathrm{F}_{6 \mathrm{NH2}, \text { obs }}-\mathrm{F}_{\text {nat, obs }}\right) \cdot$ expi $\alpha_{n a t, \text { calc }}$. For clarity, only positive density is given. The contours are drawn at $30 \%$ of the electron-density maximum of this map. The model is centered at the N6 $\alpha$ atom. Part of the FAD analogue together with chain segments around Cys58, Cys63, Lys66 and Glu201 (all labelled) is depicted, as well as parts of Tyr197, Ile198 and Arg291 (not labelled); $(+)$ water molecules
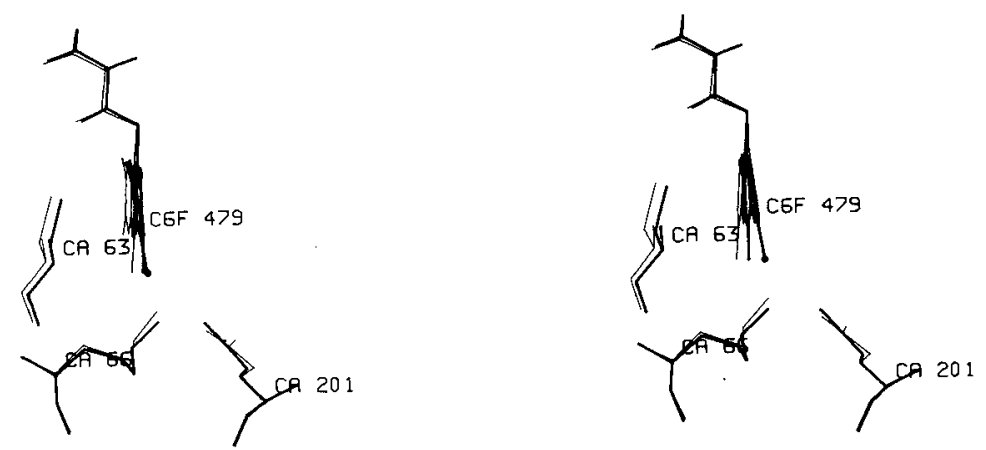

Fig. 6. Structural differences between glutathione reductase containing 6-NH $\mathrm{H}_{2}-\mathrm{FAD}$ (thick lines) and the native enzyme (thin lines). Atom $\mathrm{N} 6 \alpha$ is marked by a dot. Part of the FAD analogue together with residues Cys63, Lys66 and Glu201 is depicted

isoalloxazine follows the movement of $06 \alpha$ and tilts. The isoalloxazine tilt must involve an appreciable amount of energy because it concerns the most rigid region of the native enzyme [4].

The structure of $\mathrm{GR}\left(6-\mathrm{NH}_{2}-\mathrm{FAD}\right)$. The highest peak of the difference-Fourier map corresponds to the additional nitrogen N6 $\alpha$ (Fig. 5). Using the method described for 6-SH-FAD, the C6-N6 $\alpha$ bond length was determined as $0.15 \mathrm{~nm}$, corresponding to a single rather than a double bond [29].

As with GR(6-OH-FAD), other important peaks of the difference-Fourier map occur close to $\mathrm{O} 4 \alpha$ and $\mathrm{C} 4 \mathrm{a}$ of isoalloxazine (Fig. 5). The refinement revealed that these peaks are caused by a tilt of the isoalloxazine by almost $10^{\circ}$, similar to the tilt observed in GR(6-OH-FAD). Again, there is a substantial shift of $\mathrm{O} 4 \alpha$ related to one of the peaks; and again the disulfide bridge follows the adjacent isoalloxazine atoms resulting in a longitudinal $0.05 \mathrm{~nm}$ movement (Fig. 6).

As compared with the native enzyme, the $\mathrm{C} 6$ atom of isoalloxazine is shifted by $0.05 \mathrm{~nm}$ toward the salt bridge Lys66-Glu201. Moreover, the salt bridge is rearranged. Lys66$\mathrm{NZ}$ has moved away from $\mathrm{C} 6$, forming the closest contact to Glu201-OE1 $(0.27 \mathrm{~nm})$ instead of Glu201-OE2. The resulting distances $\mathrm{N} 6 \alpha \ldots \mathrm{NZ}, \mathrm{N} 6 \alpha \ldots \mathrm{OE} 2$ and NZ...OE2 are $0.36 \mathrm{~nm}$, $0.30 \mathrm{~nm}$ and $0.30 \mathrm{~nm}$, respectively. The large isoalloxazine tilt in a very rigid region of the native enzyme [4] indicates that the N6 $\alpha . . . O E 2$ hydrogen bond formed is rather strong. N $6 \alpha$ makes a further hydrogen bond to the water molecule Sol-89, which is shifted by $0.03 \mathrm{~nm}$ toward $\mathrm{N} 6 \alpha$ as compared to the native enzyme.

Clearly, the lengths of the distances between the new substituent and the salt bridge atoms have reversed. While the substituents $\mathrm{S} 6 \alpha$ and $06 \alpha$ are close to Lys66-NZ and about $0.05 \mathrm{~nm}$ further away from Glu201-OE2, N6 $\alpha$ is $0.06 \mathrm{~nm}$ closer to OE2 than to NZ. This observation is consistent with deprotonated S6 $\alpha$ and O6 $\alpha$ carrying a partial negative charge and therefore attracting the positive partner NZ via a hydrogen at NZ. It also agrees with two hydrogens and presumably a partial positive charge at N6 $\alpha$ which is repelled by the positively charged NZ amino group, but attracted via one of its own hydrogens to the carboxylate atom OE2.

\section{DISCUSSION}

The observed structural changes increase in the order GR(6-SH-FAD) < GR(6-OH-FAD) < GR(6- $\mathrm{NH}_{2}$-FAD). The large sulfur of 6-SH-FAD contacts Lys66-NZ at a reasonable hydrogen bond distance of $0.3 \mathrm{~nm}$ and causes almost no change. Only Gly 62 and the redoxactive disulfide bridge are pushed somewhat away. In GR(6-OH-FAD) and GR(6- $\mathrm{NH}_{2}-$ FAD) the isoalloxazine tilts and twists (Figs 4 and 6) to form hydrogen bonds between the new substituent and the adjacent salt bridge Lys66-NZ - Glu201-OE2. Most conspicuously, the $\mathrm{O} 6 \alpha$ and $N 6 \alpha$ atoms contact different partners of the salt bridge, N6 $\alpha$ causes a salt-bridge rearrangement in comparison to the native enzyme.

All data (i.e. the observed conformational changes, the determined bond lengths between $\mathrm{C} 6$ and the substituents, the protonation state at $\mathrm{pH} 7.0$ of the X-ray analyses as deduced from spectroscopically derived $\mathrm{p} K$ values) agree with a deprotonated thiol in 6-SH-FAD, a deprotonated hydroxyl in 6-OH-FAD and an amino group in 6- $\mathrm{NH}_{2}-\mathrm{FAD}$. For 6-SH- 
FAD and for 6-OH-FAD the $\mathrm{p} K$ value drops on binding to glutathione reductase, which is consistent with the stabilization of a negatively charged substituent by Lys66-NZ. A further contribution derives most likely from a delocalization of the negative charge towards N1 and $\mathrm{O} 2 \alpha$ of the isoalloxazine, where it is stabilized by the helix of amino acid residues $339-354$.

The enzyme activities of all FAD-modified enzymes analyzed are appreciably reduced (Table 1 ). The highest residual activity $(5 \%)$ is found for $\mathrm{GR}\left(6-\mathrm{NH}_{2}-\mathrm{FAD}\right)$, although this analogue causes the largest conformational changes. This indicates that catalysis is much more affected by changing the electronic properties of the isoalloxazine than by polypeptide rearrangements.

This notion is corroborated by the residual $3 \%$ enzymatic activity of GR(6-SH-FAD) as compared to no activity for GR(6-OH-FAD) as given in Table 1 . In both cases, the flavin redox potential has dropped by the conjugation of electron pairs from thiolate or hydroxylate with the isoalloxazine nucleus. While this conjugation is limited for the large thiolate atom, it is much more pronounced for the smaller hydroxylate atom giving rise to a redox potential drop of about $100 \mathrm{mV}$ [16], which renders the enzyme inactive. Using this argument, the largely reduced activity of $\mathrm{GR}\left(6-\mathrm{NH}_{2}-\mathrm{FAD}\right)$ points to a conjugation between the lone electron pair of $\mathrm{N} 6 \alpha$ and the isoalloxazine nucleus causing some decrease of the redox potential and a partial positive charge at N6 $\alpha$.

In conclusion, the described analyses of FAD-modified enzymes improved our knowledge of the chemical nature of the FAD analogues and of their interactions with functional groups of the polypeptide. The internal salt bridge Lys66Glu201 is intimately connected with protonation state and partial charge of the substituents at $\mathrm{C} 6$ of the bound flavin.

The work was supported by the Deutsche Forschungsgemeinschaft (Sfb-60/H-3, U.E. and G.E.S.) and by the US Public Health Service (grant GM 11106, V.M.).

\section{REFERENCES}

1. Williams, C. H. Jr (1976) in The enzymes (Boyer, P. D., ed.) pp. 89-173, Academic Press, New York.

2. Schirmer, R. H., Krauth-Siegel, R. L. \& Schulz, G. E. (1989) in Glutathione, part A (Dolphin, D., Poulson, R. \& Avramovic, O., eds) pp. 554-596, Wiley, New York.

3. Krauth-Siegel, R. L., Blatterspiel, R., Saleh, M., Schiltz, E., Schirmer, R. H. \& Untucht-Grau, R. (1982) Eur. J. Biochem. $121,259-267$.
4. Karplus, P. A. \& Schulz, G. E. (1987) J. Mol. Biol. 95, $701-729$.

5. Pai, E. F. \& Schulz, G. E. (1983) J. Biol. Chem. 258, 1752-1757.

6. Karplus, P. A. \& Schulz, G. E. (1989) J. Mol. Biol. 210, $163-$ 180.

7. Janes, W. \& Schulz, G. E. (1990) Biochemistry 29, 4022-4030.

8. Scrutton, N. S., Berry, A. \& Perham, R. N. (1990) Nature 343, $38-43$.

9. Ghisla, S. \& Massey, V. (1986) Biochem. J. 239, 1-12.

10. Krauth-Siegel, R. L., Schirmer, R. H. \& Ghisła, S. (1985) Eur. J. Biochem. 148, 335-344.

11. Zanetti, G., Beretta, C. \& Malandra, D. (1986) Arch. Biochem. Biophys. 244, $831-837$.

12. Massey, V., Ghisla, S., Ermler, U. \& Schulz, G. E. (1987) in Flavins and flavoproteins (Edmondson, D. E. \& McCormick, D. B., eds) pp. 79-84, de Gruyter, Berlin.

13. Ghisla, S., Massey, V. \& Yagi, K. (1986) Biochemistry 25, $3282-$ 3289.

12. Mayhew, S. G., Whitfield, C. D., Ghisla, S. \& Schuman-Jorns, M. (1974) Eur. J. Biochem. 44, 570-591.

15. Ghisla, S. \& Mayhew, S. G. (1976) Eur. J. Biochem. 63, 373390.

16. Ghisla, S. \& Mayhew, S. G. (1980) Methods Enzymol. 66, $241-$ 253.

17. Krohne-Ehrich, G., Schirmer, R. H. \& Untucht-Grau, R. (1977) Eur. J. Biochem. 17, 65-71.

18. Fritsch, K. G., Pai, E. F., Schirmer, R. H., Schulz, G. E. \& Untucht-Grau, R. (1979) Hoppe Seyler's Z. Physiol. Chem. 360, $261-262$.

19. Fritsch, K. G. (1979) Ingenieurarbeit, Fachhochschule Darmstadt.

20. Schulz, G. E., Zappe, H., Worthington, D. J. \& Rosemeyer, M. A. (1975) FEBS Lett. 54, 86-88.

21. Thieme, R., Pai, E. F., Schirmer, R. H. \& Schulz, G. E. (1981) J. Mol. Biol. 152, 763-782.

22. Jones, T. A. (1985) Methods Enzymol. 115, 157-171.

23. Tronrud, D. E., Ten Eyck, L. F. \& Matthews, B. W. (1987) Acta Crystallogr. A43, 489-501.

24. Brünger, A. T., Kuriyan, K. \& Karplus, M. (1987) Science 235, $458-460$.

25. Beinert, H. (1960) in The enzymes, 2nd edn (Boyer, P. D., Lardy, H. \& Myrbäck, K., eds) vol. 2, pp. 339-416, Academic Press, New York.

26. Massey, V., Ghisla, S. \& Yagi, K. (1986) Biochemistry 25, $8103-$ 8112.

27. Wierenga, R. K., Drenth, J. \& Schulz, G. E. (1983) J. Mol. Biol. $167,725-739$.

28. Ghisla, S., Kenney, W. C., Knappe, W. R., McIntire, W. \& Singer, P. (1980) Biochemistry 19, 2537-2544.

29. Weast, R. C., ed. (1986) CRC handbook of chemistry and physics, CRC Press, Boca Raton, Florida/USA. 